\title{
Das Schweizer Gesundheitswesen in Not?
}

\section{Lutz von Laer}

Prof. em. Dr. med., Mitglied der FMH

\author{
Ist das Schweizer Gesundheitswesen in Not? Mitnichten! Aber es steht vor einer \\ Unzahl an Problemen. Gerade aktuell kann man in den Zeitungen zahlreiche Kom- \\ mentare über das hiesige Gesundheitswesen lesen - wohl deswegen, weil der Bund \\ versucht, ins Tarifwesen einzugreifen.
}

Nahezu immer enden derartige Kommentare in einer Polemik, oft zu Ungunsten der Ärzteschaft. Dabei sind die Probleme um das Gesundheitswesen weitaus komplexer, als sie dargestellt werden, und sie können keinesfalls einer einzigen Gruppe angelastet oder gar einfach nur durch simple Sparmassnahmen gelöst werden.

Bevor man versucht, die Kosten im Gesundheitswesen «in den Griff» zu bekommen, sollte man daher zuerst eine Analyse der Situation vornehmen, um zu sehen, an welchen Punkten - wenn überhaupt - eingegriffen werden könnte:

- Im ambulanten Bereich wird nach dem TARMED abgerechnet, einem Einzelleistungstarif mit einer Zeit- und einer technischen Komponente. Jede Form eines Einzelleistungstarifes (ELT) setzt - wie schon der Name sagt - den Anreiz in die Leistung. Das bringt zwangsläufig den Verdacht mit sich, dass über das medizinisch erforderliche Mass hinaus Leistungen - aus kommerziellen Gründen - erbracht werden.

Eine nicht unerhebliche Rolle spielt der «kranke" und der "gesunde" Patient.

- Dann spielt die Verwerfung zwischen ambulanter Medizin von niedergelassenen Ärztinnen und Ärzten und ambulanter Medizin in Spitälern eine weitere grosse Rolle. Im Spital werden die dort arbeitenden Ärzte - auch wenn sie ambulante Leistungen erbringen - nach einem Zeittarif bezahlt: sie erhalten für ihre Leistungen ein Gehalt. Zudem werden die Spitäler von Gemeinden, Kantonen oder dem Bund subventioniert. Bei den Niedergelassenen handelt es sich um eigenständige Wirtschaftsunternehmen, die das wirtschaftliche Risiko ihrer Praxis selbst tragen müssen und nicht subventioniert wer- den. Sie bekommen kein Gehalt, sondern rechnen nach dem TARMED ab. Ärztliche Tätigkeit und wirtschaftliche Amortisationsgedanken müssen daher nolens volens bei den Niedergelassenen Hand in Hand gehen - im Gegensatz zu den ambulant tätigen Spitalärzten.

Die Verbesserung der Leistungsfähigkeit könnte dem Konsumverhalten übergeben werden.

- Eine nicht unerhebliche Rolle spielt der «kranke» und der "gesunde» Patient. Wir leben heute in einer begnadeten Situation, so dass wir einerseits Krankheiten mit einer bunten Vielfalt an Möglichkeiten behandeln können. Und andererseits können wir den vorhandenen, altersentsprechenden Gesundheitszustand mit einer ebensolchen Vielfalt an Möglichkeiten verbessern (Enhancement-Medizin). Dies ist absolut neu und hat uns die Alterspyramide von "gesunden» alten Menschen beschert. Sowohl für den Arzt als auch für den Patienten liegt es nahe, diese Vielfalt an Angeboten - als Mitglied einer zum Konsum verpflichteten Gesellschaft - auch als Konsumgut zu nutzen. Nicht zuletzt, nachdem die Politik wirtschaftliche Marktmechanismen in das Gesundheitswesen eingebaut hatte, in der Hoffnung auf eine wirtschaftliche Selbsregulation.

- Diese Rechnung ist nicht aufgegangen, ebenso wenig wie es durch Einführung des - schon bei der Einführung veralteten und obsoleten - DRG-Systems im stationären Bereich gelungen ist, die Kostensteigerung in den Griff zu bekommen. Ein wesentlicher Faktor für all unsere Probleme im Gesundheitswesen besteht darin, dass wir ein Versicherungsmodell - auch nach Einführung der Grundversicherung - verwenden, das hoffnungslos obsolet ist. Es 
wurde in dieser Form vor weit mehr als 100 Jahren erfunden, als es nur wenige Möglichkeiten gab, Krankheiten zu behandeln und gar keine Möglichkeiten, um die Gesundheit zu verbessern. Zu einer Zeit auch, als die meisten Menschen gar nicht alt genug wurden, um den Wunsch nach Verbesserung ihrer nachlassenden Leistungsfähigkeit verspüren zu können.

Um Transparenz und Vergleichbarkeit herzustellen, müssten bestimmte Voraussetzungen geschaffen werden:

\section{Es gilt ein Brainstorming durchzuführen, zu dem sämtliche Partner ihren Beitrag leisten müssen.}

- Auch wenn das erstaunlicherweise niemand will: aus dem Tarif für ambulante Medizin muss die Einzelleistungskomponente gestrichen und durch eine differenzierte Zeitkomponente ersetzt werden, selbst gegen den Widerstand der Akteure.

- Für die ambulante Medizin in der Praxis und im Spital müssen gleiche Voraussetzungen geschaffen werden. Das heisst, einerseits müssten die Subventionen für die Spitäler vollständig abgebaut, andererseits müssten für die niedergelassenen Ärzte vergleichbare Strukturen geschaffen werden; Strukturen, die verhindern, dass bei der täglichen Arbeit der Gedanke an die Amortisation der Praxis einfliessen kann und muss. Es existieren schon heute Strukturen wie unternehmerische Gemeinschaftspraxen, in denen den dort arbeitenden Kolleginnen und Kollegen ein Gehalt ausbezahlt wird, so dass sie nicht durch Amortisationsgedanken bei der täglichen Arbeit gestört werden.

1 Johannes Bircher und Karl-H. Wehkamp, Das ungenutzte Potential der Medizin. Rüffer \&Rub, 2006

Korrespondenz: Prof. Dr. med. Lutz von Laer Riehenstrasse 62 CH-4058 Basel lutz[at]vonlaer.ch
- Richtig schwierig wird es, wenn wir an die Beeinflussung des Patienten denken, der sich - ebenso wie der Arzt - eigentlich von einem medizinischen Konsumverhalten distanzieren sollte. Dies gilt jedoch nicht für alle Bereiche. Die Verbesserung der Leistungsfähigkeit könnte zweifelsohne dem Konsumverhalten übergeben werden, jedoch nicht die Behandlung eigentlicher Krankheiten. Also müsste man erst einmal dem Patienten eine Selbstdekla- ration anbieten, in der er sich - ohne jede wirtschaftliche Wertung - als gesund oder krank einordnen kann. Eine solche, allgemein anerkannte Unterscheidung in krank und gesund fehlt bislang. Es ist zu empfehlen, auf die von Bircher und Wehkamp $^{1}$ vorgeschlagene Definition zurückzugreifen.

- Grundlage dazu müsste in jedem Fall eine Aktualisierung des Versicherungswesens sein. Die 1996 nach einer Volksabstimmung eingeführte obligatorische Grundversicherung macht Sinn für sämtliche Arten von Erkrankungen. Sie wird aber zunehmend für Enhancement-Medizin genutzt. Das überfordert sie. Wir würden also neben der obligatorischen Kranken-Grundversicherung noch eine obligatorische Gesundheits-Grundversicherung benötigen. Dieses Unterfangen ist deshalb besonders schwierig, weil weder von einem Politiker, noch von einem Versicherer, noch von einem Arzt festgelegt werden kann, ob der Patient nun zu Lasten der einen (Krankenkasse) oder der anderen (Gesundheitskasse) behandelt werden muss. Der Entscheid dazu kann nur vom Patienten selbst getroffen werden (siehe oben), und es bedürfte einer ausgedehnteren Pilotstudie, um die Auswirkungen eines solchen Vorgehens zu überprüfen. Am einfachsten wäre es, die bisherigen Krankenkassenprämien hälftig zu teilen und den einen Teil willkürlich - und ohne jede Bewertung - als Gesundheitsprämie und den anderen Teil ebenso willkürlich als Krankheitsprämie zu registrieren.

Die Liste an Vorschlägen könnte man ziemlich ausgedehnt weiterführen. Dies würde jedoch den Rahmen des Versuches einer analytischen Aufarbeitung sprengen.

Polemik und Vorwürfe haben in der Diskussion um das Gesundheitswesen keinen Platz. Jeder der Partner wird eine vermeintliche Kröte schlucken müssen, wird aber dadurch zum Schluss mehr Vorteile einheimsen als er sich bislang erträumt hat. Es gilt also ein Brainstorming sine ira et studio durchzuführen, zu dem sämtliche Partner ihren Beitrag leisten müssen. Sie sollen dabei nicht, wie bisher üblich, für alte Modelle kämpfen, sondern neue und gemeinsame Modelle entwickeln. 